\title{
NGC 2770: High supernova rate due to interaction
}

\author{
Michał J. Michałowski ${ }^{1,2, \star}$, Christina Thöne ${ }^{3}$, Antonio de Ugarte Postigo ${ }^{3,4}$, Jens Hjorth ${ }^{4}$, Aleksandra Leśniewska ${ }^{1}$, \\ Natalia Gotkiewicz ${ }^{5}$, Wojciech Dimitrov ${ }^{1}$, Maciej P. Koprowski ${ }^{5}$, and Peter Kamphuis ${ }^{6}$ \\ 1 Astronomical Observatory Institute, Faculty of Physics, Adam Mickiewicz University, ul. Słoneczna 36, 60-286 Poznań, Poland \\ e-mail: mj . michalowski@gmail.com \\ 2 TAPIR, Mailcode 350-17, California Institute of Technology, Pasadena, CA 91125, USA \\ 3 Instituto de Astrofísica de Andalucía (IAA-CSIC), Glorieta de la Astronomía, s/n, 18008 Granada, Spain \\ ${ }^{4}$ DARK, Niels Bohr Institute, University of Copenhagen, Lyngbyvej 2, 2100 Copenhagen, Denmark \\ 5 Institute of Physics, Faculty of Physics, Astronomy and Informatics, Nicolaus Copernicus University, Grudziądzka 5, 87-100 Toruń, \\ Poland \\ ${ }^{6}$ Ruhr-Universität Bochum, Faculty of Physics and Astronomy, Astronomical Institute, 44780 Bochum, Germany
}

Received 22 June 2020 / Accepted 30 July 2020

\begin{abstract}
Context. Galaxies that hosted many core-collapse supernova (SN) explosions can be used to study the conditions necessary for the formation of massive stars. NGC 2770 was dubbed an SN factory because it hosted four core-collapse SNe in 20 years (three type Ib and one type IIn). Its star formation rate (SFR) was reported to not be enhanced and, therefore, not compatible with such a high SN rate.

Aims. We aim to explain the high SN rate of NGC 2770.

Methods. We used archival HI line data for NGC 2770 and reinterpreted the $\mathrm{H} \alpha$ and optical continuum data.

Results. Even though the continuum-based SFR indicators do not yield high values, the dust-corrected H $\alpha$ luminosity implies a high SFR, consistent with the high SN rate. Such a disparity between the SFR estimators is an indication of recently enhanced star formation activity because the continuum indicators trace long timescales of the order of $100 \mathrm{Myr}$, unlike the line indicators, which trace timescales of the order of $10 \mathrm{Myr}$. Hence, the unique feature of NGC 2770 compared to other galaxies is the fact that it was observed very shortly after the enhancement of the SFR. It also has high dust extinction, $E(B-V)$ above 1 mag. We provide support for the hypothesis that the increased SFR in NGC 2770 is due to the interaction with its companion galaxies. We report an HI bridge between NGC 2770 and its closest companion and the existence of a total of four companions within $100 \mathrm{kpc}$ (one identified for the first time). There are no clear HI concentrations close to the positions of SNe in NGC 2770 such as those detected for hosts of gamma-ray bursts (GRBs) and broad-lined SNe type Ic (IcBL). This suggests that the progenitors of type Ib SNe are not born out of recently accreted atomic gas, as was suggested for GRB and IcBL SN progenitors.
\end{abstract}

Key words. galaxies: evolution - galaxies: individual: NGC 2770 - galaxies: ISM - galaxies: star formation - supernovae: general radio lines: galaxies

\section{Introduction}

Due to their very short lifetimes, the properties and numbers of massive stars in a galaxy can be used to infer the details of star formation. Individual stars can only be detected in the most nearby galaxies, so massive stars in most galaxies can only be studied when they explode as core-collapse supernovae ( $\mathrm{SNe}$ ). In the context of using $\mathrm{SNe}$ to study star formation, it is especially promising to study galaxies with multiple SNe, as their high SN rates should be connected with conditions necessary for a given type of stellar explosion. For example, Li \& Li (1995) showed that the number of galaxies hosting multiple SNe does not deviate significantly from a Poissonian distribution, so the hosts of multiple $\mathrm{SNe}$ are not inherently different from other galaxies; they just have higher star formation rates (SFRs). Moreover, Anderson \& Soto (2013) find that galaxies with multiple $\mathrm{SNe}$ are preferentially of later morphological types, pointing to the likely connection with young stellar populations.

\footnotetext{
^ Fulbright Senior Award Fellow.
}

NGC 2770 (UGC 4806) was dubbed an SN Ib factory (Thöne et al. 2009) because it hosted three type Ib SNe in ten years. Its SFR was reported to not be enhanced and, therefore, not compatible with such a high SN rate (Thöne et al. 2009). In 2015, a fourth SN (type IIn) exploded, bringing the current rate to four over the last 20 years.

SN 1999eh was the first SN discovered in NGC 2770 (Hurst et al. 1999; Armstrong 2000) and was classified as type Ib (Jha et al. 1999). Similarly, SN 2007uy was classified as a type Ib (van der Horst et al. 2011; Roy et al. 2013) peculiar (Modjaz et al. 2014) SN. The discovery of SN 2008D (also classified as type $\mathrm{Ib}$ ) in the early phase during the observations of SN 2007uy allowed the detection of signatures of SN shock breakout (Mazzali et al. 2008; Soderberg et al. 2008; Chevalier \& Fransson 2008; Bietenholz et al. 2009; Malesani et al. 2009; Maund et al. 2009; Modjaz et al. 2009; Tanaka et al. 2009; Gorosabel et al. 2010; Couch et al. 2011).

SN 2015bh was a type IIn SN or a significant outburst of a luminous blue variable (LBV) star. It was proceeded by 20 years of outbursting activity (Elias-Rosa et al. 2016; Ofek et al. 2016; Thöne et al. 2017; Boian \& Groh 2018). 
In the single-star progenitor channel, $\mathrm{SNe}$ of both types $\mathrm{Ib} / \mathrm{c}$ (Woosley et al. 2002) and IIn (Kiewe et al. 2012) are believed to be connected with the endpoints of the evolution of the most massive stars (of the order of $>25 M_{\odot}$ ), likely Wolf-Rayet stars for the type Ib/c (Gaskell et al. 1986). On the other hand, the double-star channel for $\mathrm{SNe}$ type $\mathrm{Ib} / \mathrm{c}$ involves a less massive star $\left(8-20 M_{\odot}\right)$ with the hydrogen envelope removed through interaction in a binary system (Podsiadlowski et al. 1992). This double-star channel was also claimed for type IIb $\mathrm{SNe}$, that is to say those transitioning from type II at early times with hydrogen lines to type Ib at later times with no hydrogen lines (Woosley et al. 1987; Filippenko 1988; Filippenko et al. 1993; Nomoto et al. 1993; Krause et al. 2008; Chevalier \& Soderberg 2010).

There are a few tens of galaxies hosting multiple $\mathrm{SNe}$, but only one other galaxy has hosted more than two $\mathrm{SNe} \mathrm{Ib/c:}$ Arp 299, the interacting system of NGC 3690, and IC 694. This system hosted ten SNe (Anderson et al. 2011 and the updated list at the Open Supernova Catalog ${ }^{1}$; Guillochon et al. 2017), three of which were classified as type Ib (1998T, 2010O, and 20181rd; Li et al. 1998; Mattila et al. 2010; Perley et al. 2020), two as type IIb (SN2005U and SN2010P; Leonard \& Cenko 2005; Ryder et al. 2010), and one as type Ib/c (SN2020fkb; Tomasella et al. 2020). Similarly to NGC 2770, Arp 299 has a high SN Ib/c rate compared to the SN II rate (Anderson et al. 2011), which is common for hosts with multiple SNe (Anderson \& Soto 2013). The high SN rate of Arp 299 is consistent with its high SFR of $\sim 70 M_{\odot} \mathrm{yr}^{-1}$ (Thöne et al. 2009).

This paper has three objectives. The first is to determine whether the SFR of NGC 2770 can explain the high SN rate. The second is to test whether SNe in NGC 2770 were born in concentrations of atomic gas indicating a recent gas accretion. The third is to test whether the environment of NGC 2770 might have been responsible for its high $\mathrm{SN}$ rate.

We adopted a redshift for NGC 2770 of $z=0.00649$ (Haynes et al. 1997), a luminosity distance of $27.9 \mathrm{Mpc}$, and a corresponding scale of $0.134 \mathrm{kpc} \operatorname{arcsec}^{-1}$. This assumes a cosmological model with $H_{0}=70 \mathrm{~km} \mathrm{~s}^{-1} \mathrm{Mpc}^{-1}, \Omega_{\Lambda}=0.7$, and $\Omega_{\mathrm{m}}=0.3$.

\section{Selection and data}

The SNe in NGC 2770 were selected as part of a larger study of atomic gas in SN hosts (Gotkiewicz \& Michałowski, in prep.). We investigated all known SNe up to August 2018 with redshifts $z<0.1$ from the Open Supernova Catalog and searched the NASA/IPAC Extragalactic Database (NED) for HI data for their hosts. The study of SNe in M74 was published in Michałowski et al. (2020). The archival HI data for NGC 2770 are from Westerbork observations of neutral Hydrogen in Irregular and SPiral galaxies (WHISP; Swaters et al. 2002), taken with the Westerbork Synthesis Radio Telescope (WSRT). The data are shown in Figs. 1 and 2. The synthesised beam sizes are $24^{\prime \prime} \times 13^{\prime \prime}$, $36^{\prime \prime} \times 30^{\prime \prime}$, and $65^{\prime \prime} \times 56^{\prime \prime}$.

\section{Results}

Figure 1 shows the distribution of atomic gas in NGC 2770. There is clearly significantly more gas in the north-western than in the south-eastern part of the galaxy. The velocity field is regular and reflects a rotating disc.

\footnotetext{
https://sne.space
}

Three SNe exploded in the near or far sides of the galaxy, close to the line of sight towards the centre (see Fig. 1 of Thöne et al. 2017). SN 1999eh exploded in an atomic gas concentration visible on the HI image with the highest resolution. SN 2007uy and $2015 \mathrm{bh}$ are $\sim 15^{\prime \prime}$ ( $2 \mathrm{kpc}$ ) from the atomic gas cloud, south of the galaxy centre. However, projection effects make it impossible to securely associate $\mathrm{SNe}$ with these structures. On the other hand, SN 2008D exploded in the north-western part of the galaxy, which has more atomic gas. Its position is $\sim 30^{\prime \prime}(4 \mathrm{kpc})$ from the centre of the region with the highest HI surface density.

In Fig. 2 we show the large-scale environment of NGC 2770. The main atomic gas disc is embedded in faint emission extending $5^{\prime}(40 \mathrm{kpc})$ from the galaxy centre.

A companion galaxy called NGC $2770 \mathrm{~B}$ in Fynbo et al. (2008), Soderberg et al. (2008), and Thöne et al. (2009) is detected in $\mathrm{HI} \sim 3^{\prime}(24 \mathrm{kpc})$ to the west from the centre of NGC 2770. This galaxy was first reported by García-Ruiz et al. (2002), as KUG 0906+333A, based on the WHISP data we are using. Its velocity is clearly inconsistent with the rotation of the main disc of NGC 2770, having a negative velocity with respect to the systemic velocity, whereas the nearby north-western tip of the galaxy has a positive velocity. NGC 2770B is connected to the north-western tip of NGC 2770 by a clear HI bridge. A similar weaker feature is present on the other side of the galaxy extending east from the south-eastern tip of the galaxy. These features are labelled on Figs. 1 and 2.

To the east of NGC 2770, also 3' (24 kpc) away, GarcíaRuiz et al. (2002) reported the existence of an HI object. As they did not name it, we assigned a designation of NGC 2770C to it. It is marked on Figs. 1 and 2. In NED and the Sloan Digital Sky Survey (SDSS; York et al. 2000; Blanton et al. 2017) it is listed as GALEXASC J090946.88+330840.4 and SDSS J090946.76+330837.7, respectively. Its detections in the ultraviolet and optical imply it is also a dwarf galaxy, not just a gas cloud. The SDSS does not list a redshift for this galaxy ${ }^{2}$.

García-Ruiz et al. (2002) also reported a companion galaxy $\sim 12^{\prime}(96 \mathrm{kpc})$ to the south-east. We named this galaxy NGC 2770D. It is listed in SDSS as SDSS J091001.70+ $325659.4^{3}$ and has a spectroscopic redshift of $0.00674 \pm 0.00003$, consistent with the HI redshift and only $75 \mathrm{~km} \mathrm{~s}^{-1}$ from the redshift of NGC 2770.

Finally, we searched the $\operatorname{SDSS}^{4}$ for additional galaxies with redshifts $0.006<z<0.01$ within the WHISP dataset and found SDSS J091031.75+330329.95 at $z=0.00684829$. It is $107 \mathrm{~km} \mathrm{~s}^{-1}$ and $12.8^{\prime}$ (103 kpc; to the east) from NGC 2770. We named it NGC 2770E. It is not detected in the WHISP data.

Figure 3 shows all these nearby companions on the SDSS DR $16^{6}$ image. Table 1 shows the results of HI aperture photometry for galaxies covered by the WHISP data. For NGC 2770 we used an elliptical aperture with semiaxes of 160 and $100^{\prime \prime}$, with the angle between the semi-major axes and the north of 30 degree (towards the west). We also used two square apertures encompassing the north-western and south-eastern halves of the galaxy. For NGC 2770B, C, and D we used circular apertures with radii of $60^{\prime \prime}$. In order to estimate the HI upper

\footnotetext{
2 http://skyserver.sdss.org/DR16//en/tools/explore/ summary . aspx?id=1237664869211505073

http://skyserver.sdss.org/DR16//en/tools/explore/ summary . aspx?id=1237661384379924626

4 http://skyserver.sdss.org/dr16/en/tools/search/sql. $\operatorname{aspx}$

5 http://skyserver.sdss.org/DR16//en/tools/explore/ summary . aspx?id=1237664869211570362

6 http://skyserver.sdss.org/dr16/
} 


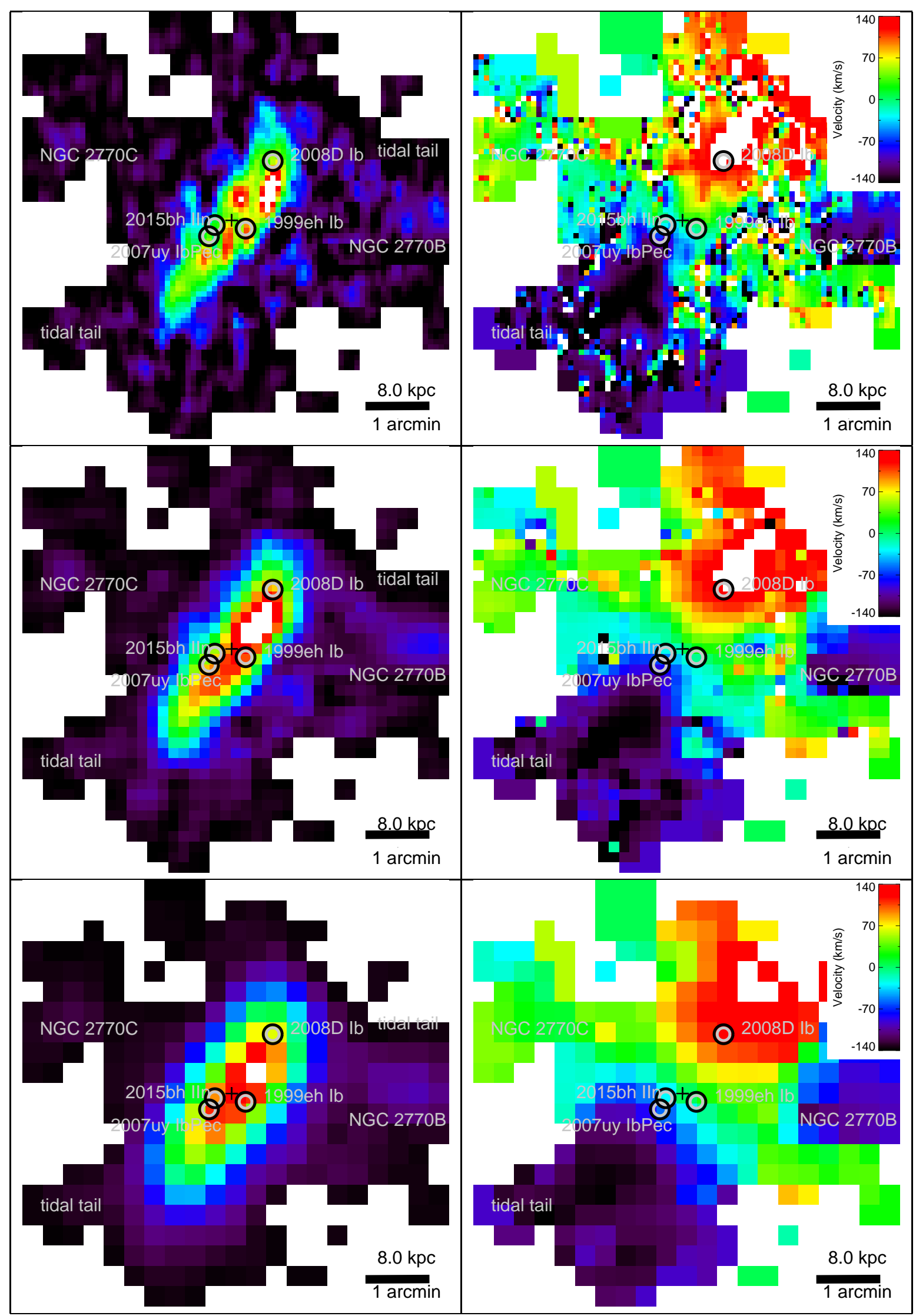

Fig. 1. Atomic gas distribution in NGC 2770. The data are at resolutions of $24^{\prime \prime} \times 13^{\prime \prime}$ (top), $36^{\prime \prime} \times 30^{\prime \prime}$ (middle), and $65^{\prime \prime} \times 56^{\prime \prime}$ (bottom). Left: zeroth moment maps (integrated emission). Right: first moment maps (velocity fields) relative to $z=0.00649\left(1945.65 \mathrm{~km} \mathrm{~s}^{-1}\right)$. The positions of $\mathrm{SNe}$ are marked by grey circles, whereas the black cross shows the position of the optical centre of the galaxy. Each panel is $6.7^{\prime}$ per side. North is up and east is to the left. NGC 2770B is at the right edge of the panels and connected to NGC 2770 by a tidal feature. NGC $2770 \mathrm{C}$ is at the left edge of the panels. 


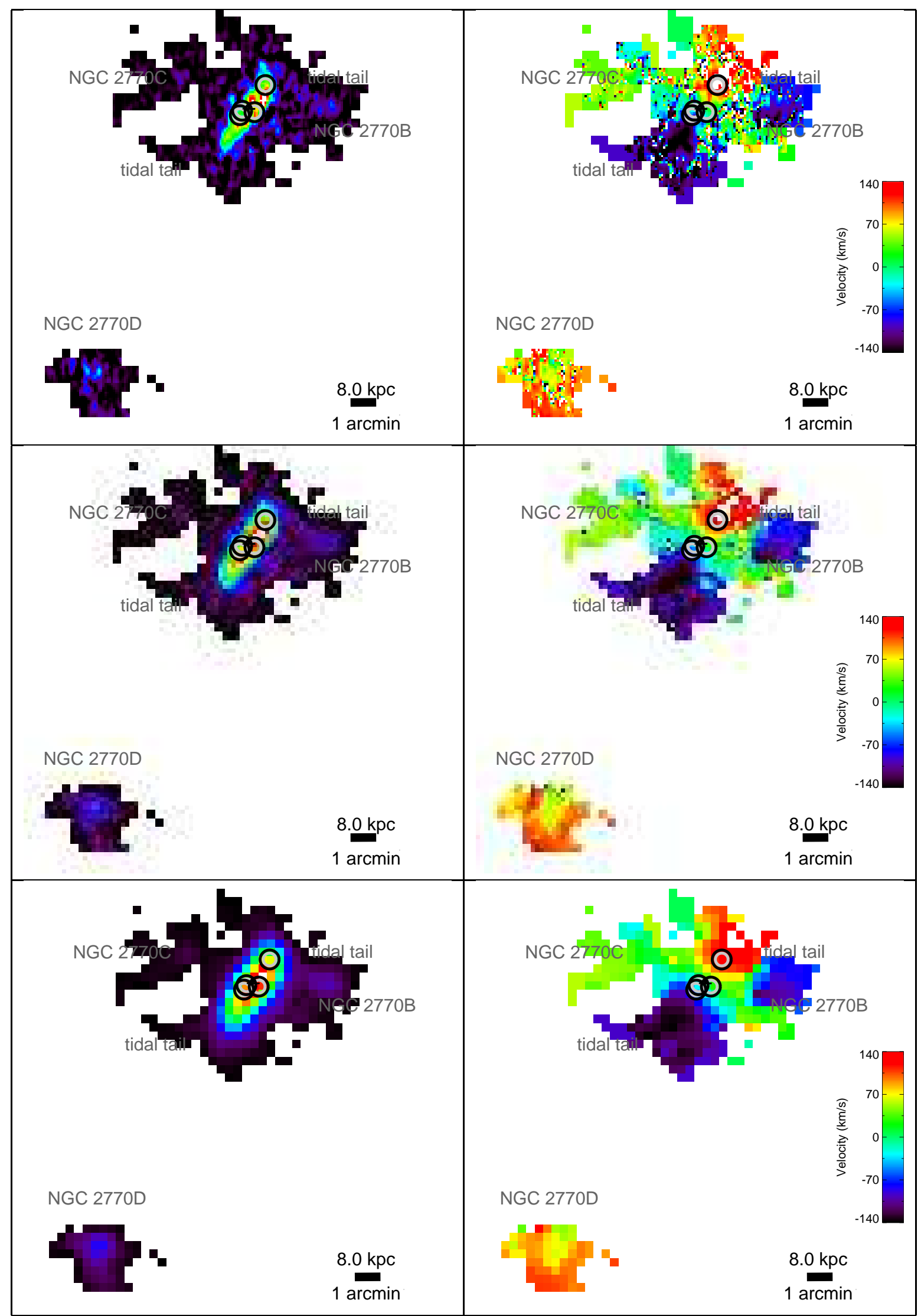

Fig. 2. As in Fig. 1, but zoomed out to show the environment of NGC 2770 . Each panel is $16.7^{\prime}$ per side. NGC $2770 \mathrm{~B}$ and C are $\sim 3^{\prime}(24 \mathrm{kpc})$ to the west and east from the centre of NGC 2770, respectively, whereas NGC 2770D is $\sim 12^{\prime}$ (96 kpc) to the south-east. There are tidal features extending from both endpoints of the NGC 2770 disc. NGC 2770E is outside the panels to the east of NGC 2770. 


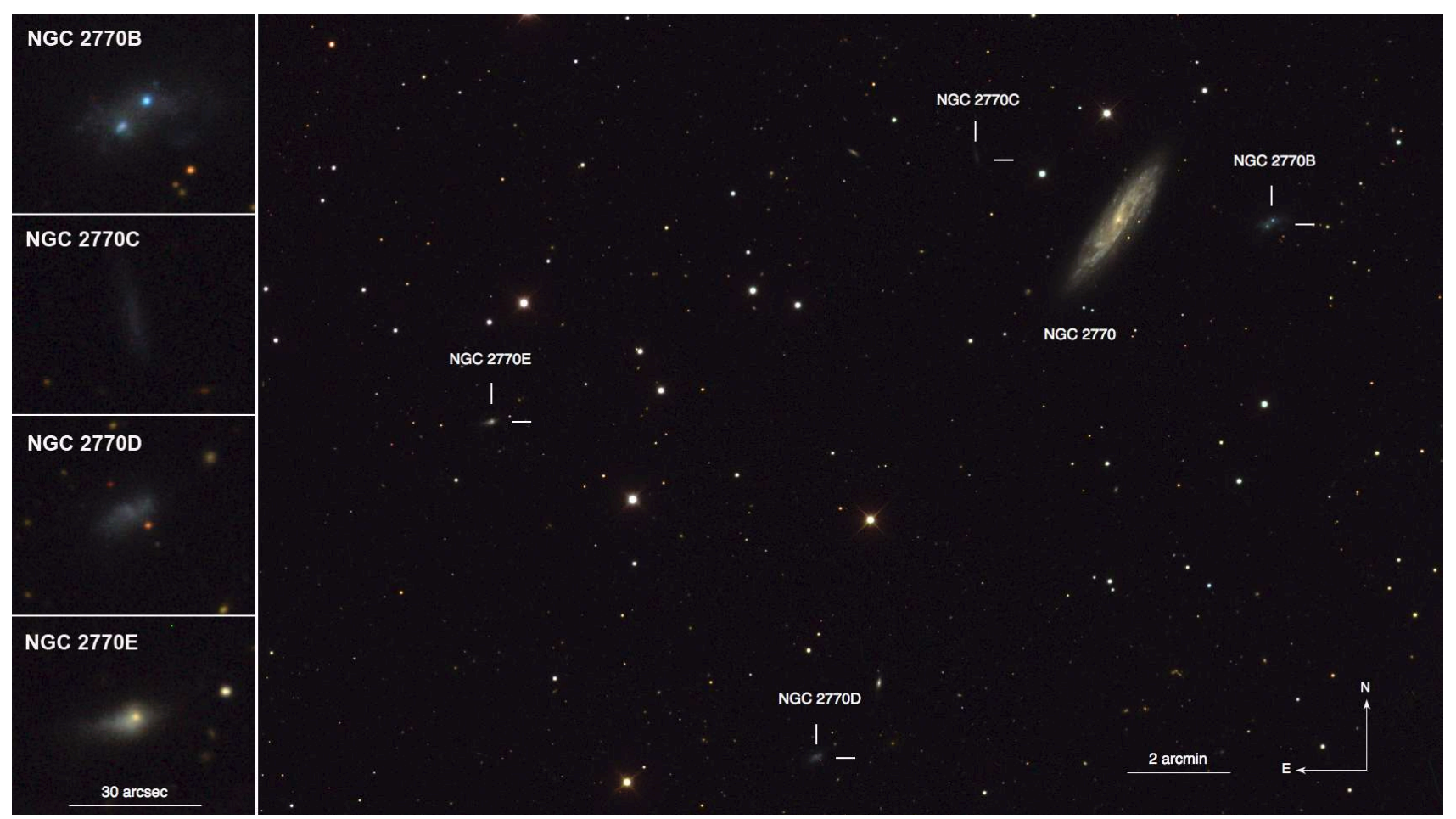

Fig. 3. Optical colour image of the NGC 2770 group with all detected companions. For the figure we use $g^{\prime}$ (blue), $r^{\prime}$ (yellow), and $i^{\prime}$ (red) filters from the SDSS DR 16 in a mosaic of 13 plates. On the left we show zoomed in images of the four companions. The different scales are indicated in the images.

Table 1. HI fluxes and atomic gas masses of galaxies in the NGC 2770 group.

\begin{tabular}{|c|c|c|c|c|c|c|c|c|c|c|}
\hline \multirow[t]{2}{*}{ Galaxy } & \multirow[t]{2}{*}{$\mathrm{RA} / \mathrm{deg}$} & \multirow[t]{2}{*}{ Dec/deg } & \multirow[t]{2}{*}{ Dist/" } & \multirow[t]{2}{*}{ Dist/kpc } & \multicolumn{3}{|c|}{ Flux $/ \mathrm{Jy} \mathrm{km} \mathrm{s}^{-1}$} & \multicolumn{3}{|c|}{$M_{\mathrm{HI}} / 10^{9} M_{\odot}$} \\
\hline & & & & & $18^{\prime \prime}$ & $33^{\prime \prime}$ & $60^{\prime \prime}$ & $18^{\prime \prime}$ & $33^{\prime \prime}$ & $60^{\prime \prime}$ \\
\hline NGC 2770 & 137.3904540 & 33.1235300 & $\cdots$ & $\ldots$ & 38.9 & 38.6 & 34.8 & 7.14 & 7.08 & 6.40 \\
\hline NGC 2770-NW & $\ldots$ & $\ldots$ & $\cdots$ & $\cdots$ & 23.0 & 22.3 & 20.2 & 4.22 & 4.10 & 3.72 \\
\hline NGC 2770-SE & $\ldots$ & $\cdots$ & $\cdots$ & $\cdots$ & 17.5 & 17.6 & 16.1 & 3.21 & 3.22 & 2.96 \\
\hline NGC 2770B & 137.3325000 & 33.1205560 & 2.9 & 23 & 2.3 & 2.2 & 1.8 & 0.43 & 0.41 & 0.34 \\
\hline NGC 2770C & 137.4448689 & 33.1438283 & 3.0 & 24 & 0.9 & 0.8 & 0.6 & 0.17 & 0.15 & 0.11 \\
\hline NGC 2770D & 137.5071249 & 32.9498569 & 12.0 & 96 & 3.4 & 3.4 & 2.7 & 0.63 & 0.62 & 0.49 \\
\hline NGC 2770E & 137.6322999 & 33.0583291 & 12.8 & 103 & $<0.3$ & $<0.3$ & $<0.4$ & $<0.05$ & $<0.05$ & $<0.08$ \\
\hline
\end{tabular}

Notes. Fluxes and masses are shown for three datasets at resolutions indicated in the header. The first row corresponds to an elliptical aperture encompassing the entire NGC 2770, whereas the following two rows correspond to rectangle apertures encompassing the north-western and southeastern halves. The fourth and fifth columns correspond to the projected distance from NGC 2770.

limit for NGC 2770E, we first calculated a typical root mean square of the cubes per nominal $20 \mathrm{~km} \mathrm{~s}^{-1}$ channel of $1.9,2.2$, and $3.1 \mathrm{mJy} \mathrm{beam}^{-1}$ for 18,33 , and $60^{\prime \prime}$ resolutions, respectively. Then we assumed that this galaxy is a point source and that its HI line spans $100 \mathrm{~km} \mathrm{~s}^{-1}$, and we reported $3 \sigma$ upper limits.

The north-western half has $25-30 \%$ more atomic gas than the south-eastern part. The total HI flux and atomic gas mass of NGC 2770 are consistent with those derived by Rhee \& van Albada $\left(1996,34 \mathrm{Jy} \mathrm{km} \mathrm{s}^{-1}\right.$ and $\left.7.04 \times 10^{9} M_{\odot}\right)$ and Matthews et al. $\left(2001,36.9 \pm 1.4 \mathrm{Jy} \mathrm{km} \mathrm{s}^{-1}\right.$ and $\left.9.76 \times 10^{9} M_{\odot}\right)$. The HI masses of NGC 2770B and D are around 20 times lower than that of NGC 2770, whereas NGC 2770C has 40 times less atomic gas than NGC 2770. NGC 2770E has at least 100 times less atomic gas than NGC 2770 .

We also investigated galaxies further away from NGC 2770 that are not covered by the WHISP data. Within $60^{\prime}(482 \mathrm{kpc})$ and $\pm 500 \mathrm{~km} \mathrm{~s}^{-1}$ of NGC 2770 (velocity of $1945.65 \mathrm{~km} \mathrm{~s}^{-1}$ ),
NED lists four galaxies: NGC 2770B, NGC 2770D, and WISEA J091312.36+332639.2 $50^{\prime}(400 \mathrm{kpc})$ to the northeast at a redshift of $0.006047\left(1813 \mathrm{~km} \mathrm{~s}^{-1}\right)$, and WISEA $\mathrm{J} 090706.29+322219.5^{8}, 55^{\prime}(440 \mathrm{kpc})$ to the south-west at a redshift of $0.005814\left(1743 \mathrm{~km} \mathrm{~s}^{-1}\right)$. The SDSS does not list any additional galaxies within these search criteria. Moreover, García-Ruiz et al. (2002, their Table 7) reported three other galaxies within 100' (800 kpc) of NGC 2770: IC 2445 (91.4', $735 \mathrm{kpc}$ ), CG 0010 (96.1', $773 \mathrm{kpc}$ ), and UGC 04777 (96.7', $777 \mathrm{kpc}$ ). NGC 2770 is not covered by the Arecibo Legacy Fast

7 Or SDSS J091312.37+332640.2 at $z=0.00607566$;

http://skyserver.sdss.org/DR16//en/tools/explore/ summary . aspx?id=1237664869748637939

8 Or SDSS J090706.26+322219.4 at $z=0.006475154$

http://skyserver.sdss.org/DR16//en/tools/explore/ summary . aspx?id=1237661126154125569 
ALFA Survey (ALFALFA; Haynes et al. 2018), prohibiting a systematic HI search of companions.

\section{Discussion}

\subsection{Enhanced SFR explaning high $S N$ rate}

We now present the evidence that the SFR of NGC 2770 has been recently enhanced, which explains the high $\mathrm{SN}$ rate. The baseline non-enhanced SFR can be calculated in several ways. The mainsequence SFR for a stellar mass of $2 \times 10^{10} M_{\odot}$ (Thöne et al. 2009) at $z=0.00649$ is $1.1 M_{\odot} \mathrm{yr}^{-1}$ (Speagle et al. 2014). The atomic gas mass $M_{\mathrm{HI}}=7.14 \times 10^{9} M_{\odot}$ (Table 1) predicts SFR = $1.9 M_{\odot} \mathrm{yr}^{-1}$ Michałowski et al. (2015, Eq. (1)). Finally, the total molecular gas mass of $\log \left(M_{\mathrm{H} 2} / M_{\odot}\right)=8.9$ (van der Horst et al. 2011) predicts $\mathrm{SFR}=0.8 M_{\odot} \mathrm{yr}^{-1}$ Michałowski et al. (2018b, Eq. (1)).

On the other hand, the level of the expected enhanced SFR can be estimated from the measured SN rate $\left(R_{\mathrm{SN}}\right)$. The scaling of Strolger et al. (2015) of $R_{\mathrm{SN}}\left(\mathrm{SN} \mathrm{yr}^{-1}\right)=0.01 \times \operatorname{SFR}\left(M_{\odot} \mathrm{yr}^{-1}\right)$ as well as four SNe in 20 years imply SFR $=20 M_{\odot} \mathrm{yr}^{-1}$. This calibration takes into account all $\mathrm{SNe}$ (i.e. progenitors masses larger than $8 M_{\odot}$ ). NGC 2770 hosted SNe type Ib and IIn, so, if their progenitors were more massive, the SFR required to explain their frequency would be higher. On the other hand, a double-star channel progenitor predicts lower masses (Podsiadlowski et al. 1992), consistent with the range analysed by Strolger et al. (2015).

The SFR of NGC 2770 measured from continuum estimators not affected by dust attenuation (infrared, radio, and full spectral energy distribution modelling) is SFR $=1.1-1.2 M_{\odot} \mathrm{yr}^{-1}$ (Thöne et al. 2009). On the other hand, the dust-uncorrected ultraviolet SFR was measured to be $0.5 M_{\odot} \mathrm{yr}^{-1}$ (Thöne et al. 2009). The comparison with the radio estimate suggests a low level of dust attenuation of $A_{\mathrm{UV}}=2.5 \log \left(\mathrm{SFR}_{\text {radio }} / \mathrm{SFR}_{\mathrm{UV}}\right)=0.95 \mathrm{mag}$ (Michałowski et al. 2012a) or $A_{V}=A_{\mathrm{UV}} / 2.2=0.43 \mathrm{mag}$, assuming a Small Magellanic Cloud extinction curve (Gordon et al. 2003). These low values do not imply that the SFR is enhanced. However, if the enhancement is recent (of the order of $10 \mathrm{Myr}$ ), then these SFR estimates are not expected to increase because these indicators trace timescales of the order of $100 \mathrm{Myr}$. In the case of a recent enhancement, only the SFRs from line indicators $(\mathrm{H} \alpha,[\mathrm{OII}],[\mathrm{CII}])$ are expected to increase because they trace much shorter timescales of the order of $10 \mathrm{Myr}$.

Based on the $\mathrm{H} \alpha$ narrowband filter observations, Thöne et al. (2009) reported a total SFR of $0.42 M_{\odot} \mathrm{yr}^{-1}$ without dust correction. The median and mean colour excess measured in regions of NGC 2770 are $E(B-V)=1.64 \pm 0.24$ and $1.67 \pm 0.15 \mathrm{mag}$, respectively (Thöne et al. 2009). This attenuation level is much higher than that experienced by the continuum emission. We assumed the slope of the attenuation curve $R_{V} \equiv A_{V} / E(B-V)=$ 3.1 and that the extinction at the $V$-band $\left(A_{V}\right)$ is similar to that at the wavelength of the $\mathrm{H} \alpha$ line (Gordon et al. 2003; Michałowski et al. 2012b). This corresponds to dust-corrected $\mathrm{H} \alpha$ SFR of $45.4 \pm 0.8$ and $49.1 \pm 0.7 M_{\odot} \mathrm{yr}^{-1}$.

These high values indicate a significant enhancement in star formation activity, and they can fully explain the high SN rate in NGC 2770. The merging system Arp 299 does not resemble the morphology of NGC 2770, but it has a similarly high SFR (Thöne et al. 2009), and its high $\mathrm{SN} \mathrm{Ib/c} \mathrm{rate} \mathrm{was} \mathrm{also} \mathrm{claimed}$ to be a result of the young age of the most recent star formation episode (Anderson et al. 2011).

The SFR enhancement in NGC 2770 must have happened recently (of the order of $10 \mathrm{Myr}$ ago) because the SFRs based on the infrared and radio have not yet had time to increase. High line-to-continuum SFR ratios were also reported for gamma-ray burst (GRB) hosts and interpreted as recently enhanced star formation activity (Michałowski et al. 2016).

Hence, the unique feature of NGC 2770 compared to other galaxies is the fact that it was observed very shortly after the enhancement of the SFR. It also has high dust extinction: $E(B-V)$ above 1 mag is very rare and is found only in galaxies with SFRs of a few tens of $M_{\odot} \mathrm{yr}^{-1}$ (Kewley et al. 2006; Wijesinghe et al. 2011; Kreckel et al. 2013; Xiao et al. 2012; Catalán-Torrecilla et al. 2015; Davies et al. 2016; Wang et al. 2016).

Other host galaxies of multiple $\mathrm{SNe}$ type $\mathrm{Ib} / \mathrm{c}$ also have sufficiently high SFRs to explain the measured SN rate. In the sample of galaxies having hosted many SNe compiled by Thöne et al. (2009), apart from NGC 2770, there are six other galaxies with two $\mathrm{SNe}$ type $\mathrm{Ib} / \mathrm{c}^{9}$. Out of the five with measured radio SFRs, three have high values of $6-70 M_{\odot} \mathrm{yr}^{-1}$. NGC 3810 and NGC 7714 have radio SFRs around $1 M_{\odot} \mathrm{yr}^{-1}$, but their SFRs measured with the $\mathrm{H} \alpha$ line are a few times higher, 4-6 $M_{\odot} \mathrm{yr}^{-1}$ (James et al. 2004). That implies that their SFRs have been recently enhanced, like that of NGC 2770. Two SNe in 20 years would imply an expected SFR of $10 M_{\odot} \mathrm{yr}^{-1}$ (as above from the calibration of Strolger et al. 2015). Hence, within likely errors in this calibration, these SFRs are consistent with the measured SN rate. We note that out of the six galaxies that hosted more than one $\mathrm{SN} \mathrm{Ib/c}$, only two (NGC 3464 and 3810) have undisturbed spiral morphology similar to that of NGC 2770. The remaining four are strongly interacting systems with strong disturbances.

\subsection{Interaction resulting in an enhanced SFR}

The HI bridge between NGC 2770 and NGC 2770B provides definitive proof that they are interacting. This was suggested by Fynbo et al. (2008), Soderberg et al. (2008), and Thöne et al. (2009), but they did not find any obvious perturbations in the optical images of NGC 2770. This highlights the importance of HI observations in looking for interaction signatures. The bridge, together with the feature on the opposite side of NGC 2770 (at the south-eastern tip of the galaxy), form a characteristic tidal feature that is always seen in merger simulations (Hopkins et al. 2006; Hayward et al. 2012, 2014; Pettitt et al. 2016; Oh et al. 2015). The HI asymmetry of the main disc in NGC 2770 is also consistent with this scenario. There is also more molecular gas in the north-western part of the galaxy (van der Horst et al. 2011), so the HI deficit in the south-eastern part is not due to more rapid conversion to the molecular phase. NGC 2770 may also be interacting with NGC 2770C, D, and E, but this needs to be tested with deeper HI observations. The interaction with NGC 2770C is especially likely given its proximity to NGC 2770 .

The interaction with these galaxies might have led to enhanced star formation activity, which would explain the high SN rate in NGC 2770. This is consistent with the study of galaxies hosting several SNe implying that a significant fraction of those hosting SN type $\mathrm{Ib} / \mathrm{c}$ are classified as starbursts, irregular, or interacting, unlike those hosting SNe type II (Thöne et al. 2009). Similarly, in the sample of SN hosts analysed by Galbany et al. (2018), SNe type $\mathrm{Ib} / \mathrm{c}$ are associated with regions with the highest SFRs (their Figs. 5 and 9). Moreover, Arabsalmani et al. $(2015,2019)$ provided evidence of an on-going interaction for a GRB host. This is likely because

9 NGC 3690, NGC 2207, NGC 3810, NGC 7714, NGC 4568, NGC 3464. 
progenitors of SN type Ib/c and GRBs are more massive stars, so they are found preferentially in galaxies with recent enhancement of star formation.

In the previous section we showed that in order to explain the discrepancy between the SFRs based on continuum and line estimators, a recent enhancement of the SFR in NGC 2770 is needed. Indeed, simulations of interacting galaxies show that SFRs rise on the timescale of a few tens of Myr or quicker (Hayward et al. 2014; Fensch et al. 2017).

\subsection{Environment of the $\mathrm{SNe}$}

The scenario of a recently enhanced SFR implies that the SNe in NGC 2770 are born in recently formed dusty star-forming regions. Indeed, these $\mathrm{SNe}$ were reported to be significantly affected by dust. Roy et al. (2013) reported $E(B-V)=0.63 \mathrm{mag}$ for SN 2007uy; Malesani et al. (2009) reported $E(B-V)=$ $0.8 \mathrm{mag}$ for SN 2008D and Thöne et al. (2017) measured high pre-explosion Balmer decrement for SN 20015bh, which implies $E(B-V)=0.93 \mathrm{mag}$.

It is unclear if the SNe in NGC 2770 are associated with high atomic gas surface density regions. They appear to be close to peaks of HI distributions, but only SN 1999eh exploded within such concentration. Moreover, SN 2008D exploded close to the highest HI peak in the galaxy.

Hence, type Ib SNe have different environments than GRBs and broad-lined SNe type Ic (IcBL), which were shown to happen close to significant asymmetric off-centre HI concentrations (Michałowski et al. 2015, 2016, 2018a, 2020; Arabsalmani et al. 2015, 2019). This suggests that the progenitors of type $\mathrm{Ib} \mathrm{SNe}$ are not born out of recently accreted atomic gas, as was suggested for GRB and IcBL SN progenitors ${ }^{10}$.

\subsection{Rotation}

van der Horst et al. (2011) concluded that the rotation of the $\mathrm{CO}$ disc (high velocities in the north-western part; see their Fig. 4) is opposite to the HI rotation. They referred to the position-velocity diagram of Rhee \& van Albada (1996, their Fig. 4), which shows low velocities in the north-western part. However, we believe that the galaxy sides are mis-labelled on Fig. 4 of Rhee \& van Albada (1996) because the WHISP data show high velocities in the north-western part (Fig. 1), consistent with the CO rotation. Moreover, Fig. 4 of Rhee \& van Albada (1996) shows that the galaxy side with more atomic gas (labelled SE there) has high velocities, so it should correspond to the north-western part. Hence, the conclusion of van der Horst et al. (2011) that the CO emission in NGC 2770 is a result of a recent merger with a dwarf is likely incorrect. NGC 2770 is currently interacting with its companion, but there is no evidence for a recent merger. We also note that the counter-rotating component at low velocities $\left(2^{\prime}\right.$ north at $-100 \mathrm{~km} \mathrm{~s}^{-1}$ ), which van der Horst et al. (2011) noticed in the data of Rhee \& van Albada (1996), is NGC 2770B.

\section{Conclusions}

NGC 2770 has hosted three type Ib and one type IIn SNe over the last 20 years. This high SN rate has not been fully explained

\footnotetext{
${ }^{10}$ Only AT 2018cow, with an unclear nature, did not happen in an offcentre asymmetric Hi feature (Michałowski et al. 2019) and possibly only close to a gas ring (Roychowdhury et al. 2019).
}

and is not compatible with the previously reported SFR. We used archival HI line data for NGC 2770 and reinterpreted the $\mathrm{H} \alpha$ and optical continuum data.

Even though the continuum-based SFR indicators do not yield high values, the dust-corrected $\mathrm{H} \alpha$ luminosity implies a high SFR, consistent with the high SN rate. Such a disparity between the SFR estimators is an indication of recently enhanced star formation activity because the continuum indicators trace long timescales of the order of $100 \mathrm{Myr}$, unlike the line indicators, which trace timescales of the order of $10 \mathrm{Myr}$.

Hence, the unique feature of NGC 2770 compared to other galaxies is the fact that it was observed very shortly after the enhancement of the SFR. It also has high dust extinction, $E(B-$ $V$ ) above $1 \mathrm{mag}$.

We provide support for the hypothesis that the increased SFR in NGC 2770 is due to the interaction with its companion galaxies. The HI bridge between NGC 2770 and NGC 2770B is definitive proof that they are interacting. The atomic disc of NGC 2770 is also asymmetric. The interaction is not obvious on the optical image, which does not reveal disturbances. We report the existence of a total of four companions within $100 \mathrm{kpc}$ (one identified for the first time).

Supernovae in NGC 2770 exploded close to regions rich in atomic gas, but there are no HI concentrations as dramatic as those detected for hosts of GRBs and type IcBL SNe. This suggests that the progenitors of type $\mathrm{Ib} \mathrm{SNe}$ are not born out of recently accreted atomic gas, as was suggested for GRB and IcBL SN progenitors.

Acknowledgements. We wish to thank the referee, Artur Hakobyan, for helpful suggestions and for pointing out the existence of NGC 2770C and E, and Joanna Baradziej for discussion and comments. M.J.M. acknowledges the support of the National Science Centre, Poland through the SONATA BIS grant 2018/30/E/ST9/00208, and of the Polish-U.S. Fulbright Commission. A.d.U.P. and C.C.T. acknowledge the support from Ramón y Cajal fellowships (RyC-2012-09975 and RyC-2012-09984) and from the Spanish research project AYA2017-89384-P. A.L. acknowledges the support of the National Science Centre, Poland through the SONATA BIS grant 2018/30/E/ST9/00208. J.H. was supported by a VILLUM FONDEN Investigator grant (project number 16599). M.P.K. acknowledges support from the First TEAM grant of the Foundation for Polish Science No. POIR.04.04.00-00-5D21/18-00. P.K. is partially supported by the BMBF project 05A17PC2 for D-MeerKAT. The WSRT is operated by the Netherlands Foundation for Research in Astronomy with financial support from the Netherlands Organization for Scientific Research (NWO). Funding for the SDSS and SDSS-II has been provided by the Alfred P. Sloan Foundation, the Participating Institutions, the National Science Foundation, the U.S. Department of Energy, the National Aeronautics and Space Administration, the Japanese Monbukagakusho, the Max Planck Society, and the Higher Education Funding Council for England. The SDSS Web Site is http://www . sdss . org/. The SDSS is managed by the Astrophysical Research Consortium for the Participating Institutions. The Participating Institutions are the American Museum of Natural History, Astrophysical Institute Potsdam, University of Basel, University of Cambridge, Case Western Reserve University, University of Chicago, Drexel University, Fermilab, the Institute for Advanced Study, the Japan Participation Group, Johns Hopkins University, the Joint Institute for Nuclear Astrophysics, the Kavli Institute for Particle Astrophysics and Cosmology, the Korean Scientist Group, the Chinese Academy of Sciences (LAMOST), Los Alamos National Laboratory, the Max-Planck-Institute for Astronomy (MPIA), the MaxPlanck-Institute for Astrophysics (MPA), New Mexico State University, Ohio State University, University of Pittsburgh, University of Portsmouth, Princeton University, the United States Naval Observatory, and the University of Washington. We acknowledge the usage of the HyperLeda database (http: //leda.univ-lyon1.fr). This research has made use of the Open Supernova Catalog (https://sne.space); NASA/IPAC Extragalactic Database (NED) which is operated by the Jet Propulsion Laboratory, California Institute of Technology, under contract with the National Aeronautics and Space Administration; SAOImage DS9, developed by Smithsonian Astrophysical Observatory (Joye et al. 2003); Edward Wright cosmology calculator (Wright 2006); the WebPlotDigitizer of Ankit Rohatgi (arohatgi . info/WebPlotDigitizer) and NASA's Astrophysics Data System Bibliographic Services. 


\section{References}

Anderson, J. P., \& Soto, M. 2013, A\&A, 550, A69

Anderson, J. P., Habergham, S. M., \& James, P. A. 2011, MNRAS, 416, 567

Arabsalmani, M., Roychowdhury, S., Zwaan, M. A., Kanekar, N., \& Michałowski, M. J. 2015, MNRAS, 454, L51

Arabsalmani, M., Roychowdhury, S., Starkenburg, T. K., et al. 2019, MNRAS, 485,5411

Armstrong, M. W. 2000, J. Br. Astron. Assoc., 110, 54

Bietenholz, M. F., Soderberg, A. M., \& Bartel, N. 2009, ApJ, 694, L6

Blanton, M. R., Bershady, M. A., Abolfathi, B., et al. 2017, AJ, 154, 28

Boian, I., \& Groh, J. H. 2018, A\&A, 617, A115

Catalán-Torrecilla, C., Gil de Paz, A., Castillo-Morales, A., et al. 2015, A\&A, 584, A87

Chevalier, R. A., \& Fransson, C. 2008, ApJ, 683, L135

Chevalier, R. A., \& Soderberg, A. M. 2010, ApJ, 711, L40

Couch, S. M., Pooley, D., Wheeler, J. C., \& Milosavljević, M. 2011, ApJ, 727 104

Davies, L. J. M., Driver, S. P., Robotham, A. S. G., et al. 2016, MNRAS, 461, 458

Elias-Rosa, N., Pastorello, A., Benetti, S., et al. 2016, MNRAS, 463, 3894

Fensch, J., Renaud, F., Bournaud, F., et al. 2017, MNRAS, 465, 1934

Filippenko, A. V. 1988, AJ, 96, 1941

Filippenko, A. V., Matheson, T., \& Ho, L. C. 1993, ApJ, 415, L103

Fynbo, J. P. U., Malesani, D., Augusteijn, T., \& Niemi, S. M. 2008, GRB Coord Netw., 7186, 1

Galbany, L., Anderson, J. P., Sánchez, S. F., et al. 2018, ApJ, 855, 107

García-Ruiz, I., Sancisi, R., \& Kuijken, K. 2002, A\&A, 394, 769

Gaskell, C. M., Cappellaro, E., Dinerstein, H. L., et al. 1986, ApJ, 306, L77

Gordon, K. D., Clayton, G. C., Misselt, K. A., Landolt, A. U., \& Wolff, M. J. 2003, ApJ, 594, 279

Gorosabel, J., de Ugarte Postigo, A., Castro-Tirado, A. J., et al. 2010, A\&A, 522, A14

Guillochon, J., Parrent, J., Kelley, L. Z., \& Margutti, R. 2017, ApJ, 835, 64

Haynes, M. P., Giovanelli, R., Herter, T., et al. 1997, AJ, 113, 1197

Haynes, M. P., Giovanelli, R., Kent, B. R., et al. 2018, ApJ, 861, 49

Hayward, C. C., Jonsson, P., Kereš, D., et al. 2012, MNRAS, 424, 951

Hayward, C. C., Torrey, P., Springel, V., Hernquist, L., \& Vogelsberger, M. 2014, MNRAS, 442, 1992

Hopkins, P. F., Hernquist, L., Cox, T. J., et al. 2006, ApJS, 163, 1

Hurst, G. M., Armstrong, M., \& Boles, T. 1999, IAU Circ., 7282, 1

James, P. A., Shane, N. S., Beckman, J. E., et al. 2004, A\&A, 414, 23

Jha, S., Garnavich, P., Challis, P., Kirshner, R., \& Calkins, M. 1999, IAU Circ. 7282,2

Joye, W. A., \& Mandel, E. 2003, in Astronomical Data Analysis Software and Systems XII, eds. H. E. Payne, R. I. Jedrzejewski, \& R. N. Hook, ASP Conf. Ser., 295, 489

Kewley, L. J., Groves, B., Kauffmann, G., \& Heckman, T. 2006, MNRAS, 372, 961

Kiewe, M., Gal-Yam, A., Arcavi, I., et al. 2012, ApJ, 744, 10

Krause, O., Birkmann, S. M., Usuda, T., et al. 2008, Science, 320, 1195

Kreckel, K., Groves, B., Schinnerer, E., et al. 2013, ApJ, 771, 62
Leonard, D. C., \& Cenko, S. B. 2005, ATel, 431, 1

Li, W., \& Li, Z. 1995, A\&A, 301, 666

Li, W. D., Li, C., Wan, Z., et al. 1998, IAU Circ., 6830, 1

Malesani, D., Fynbo, J. P. U., Hjorth, J., et al. 2009, ApJ, 692, L84

Matthews, L. D., van Driel, W., \& Monnier-Ragaigne, D. 2001, A\&A, 365, 1

Mattila, S., Kankare, E., Datson, J., \& Pastorello, A. 2010, Cent. Bur. Electron. Telegrams, 2149, 1

Maund, J. R., Wheeler, J. C., Baade, D., et al. 2009, ApJ, 705, 1139

Mazzali, P. A., Valenti, S., Della, Valle M., et al. 2008, Science, 321, 1185

Michałowski, M. J., Dunlop, J. S., Cirasuolo, M., et al. 2012a, A\&A, 541, A85

Michałowski, M. J., Kamble, A., Hjorth, J., et al. 2012b, ApJ, 755, 85

Michałowski, M. J., Gentile, G., Hjorth, J., et al. 2015, A\&A, 582, A78

Michałowski, M. J., Castro Cerón, J. M., Wardlow, J. L., et al. 2016, A\&A, 595, A72

Michałowski, M. J., Karska, A., Rizzo, J. R., et al. 2018a, A\&A, 617, A143

Michałowski, M. J., Gentile, G., Krühler, T., et al. 2018b, A\&A, 618, A104

Michałowski, M. J., Kamphuis, P., Hjorth, J., et al. 2019, A\&A, 627, A106

Michałowski, M. J., Gotkiewicz, N., Hjorth, J., \& Kamphuis, P. 2020, A\&A, 638, A47

Modjaz, M., Blondin, S., Kirshner, R. P., et al. 2014, AJ, 147, 99

Modjaz, M., Li, W., Butler, N., et al. 2009, ApJ, 702, 226

Nomoto, K., Suzuki, T., Shigeyama, T., et al. 1993, Nature, 364, 507

Ofek, E. O., Cenko, S. B., Shaviv, N. J., et al. 2016, ApJ, 824, 6

Oh, S. H., Kim, W. T., \& Lee, H. M. 2015, ApJ, 807, 73

Perley, D. A., Taggart, K., Dahiwale, A., \& Fremling, C. 2020, Transient Name Server Classification Report, 2020-1155, 1

Pettitt, A. R., Tasker, E. J., \& Wadsley, J. W. 2016, MNRAS, 458, 3990

Podsiadlowski, P., Joss, P. C., \& Hsu, J. J. L. 1992, ApJ, 391, 246

Rhee, M. H., \& van Albada, T. S. 1996, A\&AS, 115, 407

Roy, R., Kumar, B., Maund, J. R., et al. 2013, MNRAS, 434, 2032

Roychowdhury, S., Arabsalmani, M., \& Kanekar, N. 2019, MNRAS, 485, L93

Ryder, S., Mattila, S., Kankare, E., \& Perez-Torres, M. 2010, Cent. Bur. Electron. Telegrams, 2189, 1

Soderberg, A. M., Berger, E., Page, K. L., et al. 2008, Nature, 453, 469

Speagle, J. S., Steinhardt, C. L., Capak, P. L., \& Silverman, J. D. 2014, ApJS, 214,15

Strolger, L. G., Dahlen, T., Rodney, S. A., et al. 2015, ApJ, 813, 93

Swaters, R. A., van Albada, T. S., van der Hulst, J. M., \& Sancisi, R. 2002, A\&A, 390,829

Tanaka, M., Tominaga, N., Nomoto, K., et al. 2009, ApJ, 692, 1131

Thöne, C. C., Michałowski, M. J., Leloudas, G., et al. 2009, ApJ, 698, 1307

Thöne, C. C., de Ugarte Postigo, A., Leloudas, G., et al. 2017, A\&A, 599, A129

Tomasella, L., Benetti, S., Ochner, P., et al. 2020, ATel, 13615, 1

van der Horst, A. J., Kamble, A. P., Paragi, Z., et al. 2011, ApJ, 726, 99

Wang, L., Norberg, P., Gunawardhana, M. L. P., et al. 2016, MNRAS, 461, 1898

Wijesinghe, D. B., da Cunha, E., Hopkins, A. M., et al. 2011, MNRAS, 415, 1002

Woosley, S. E., Pinto, P. A., Martin, P. G., \& Weaver, T. A. 1987, ApJ, 318, 664

Woosley, S. E., Heger, A., \& Weaver, T. A. 2002, Rev. Mod. Phys., 74, 1015

Wright, E. L. 2006, PASP, 118, 1711

Xiao, T., Wang, T., Wang, H., et al. 2012, MNRAS, 421, 486

York, D. G., Adelman, J., Anderson, J., John, E., et al. 2000, AJ, 120, 1579 